Theories \& Applications the International Edition

Printed Version: (ISSN 2090-5262)

Online Version: (ISSN 2090-5270)

November 2011, Volume 1, No. 3 Pages (1 -15)

\title{
Studying the Distinctive Biomechanical Indicators for Comparative Three Levels ofPerformance of Salto Backward Biked on Balance Beam
}

\author{
Mohamed GaberBrekaa *, Mervet Ahmed Kamal **
}

The current research aims at identifying the distinctive indicators of the comparative biomechanical efficiency for the Salto backward biked on balance beam during three levels of performance. The researcher used the experimental approach (one-group deign) through follow-up measurements. Sample (3 junior female gymnastics performers - 11-12 old year phase) was purposefully chosen from the Military Institution Sports Club in Alexandria - Egypt. They were all beginners in learning the Salto backward biked skills. The researchers concluded the following distinctive biomechanical indicators to differentiated among the three three levels of performance (preliminary conformity - good conformity-master): Maximum fade out distinctive indicators are upper arm-forearm angle, horizontal acceleration, horizontal strength and resultant strength. Feet-off the beam distinctive indicators are foot-leg angle and resultant momentum. Flight and circling distinctive indicators are upper arm-trunk angle, thigh-leg angle, horizontal acceleration and resultant momentum. Feet-touching-the beam distinctive indicators are foot-leg angle and horizontal momentum. Stability and balance distinctive indicators are thigh-trunk angle, vertical momentum and resultant acceleration. Researchers also concluded the duration of performance for final phase and a predictive equations indicating the performance level of the Salto backward biked on the balance beam during three levels of performance

Key words: biomechanical efficiency - distinctive indicators three levels ofperformance - Salto backward biked-gymnastics.

\section{Introduction:}

$\mathbf{S}^{\mathrm{s}}$ ports movements are studied and evaluated physiological and psychological. The biomechanical aspect is one of the most important aspects as it is widely used in most research and scientific references (1).

Biomechanical study of sports movements is an objective means of evaluating improving and modifying technical performance as it uses objective means of evaluation. As a field of science, biomechanics aims at understanding technical performance using various scientificsystems and procedures to improve and develop it, along with directing the learning and training processes to achieve elite performance levels(2).

\footnotetext{
*Professor and Head of Biomechanics Department, The Faculty of Physical Education, Tanta University, Egypt.

**Assistant Professor, Biomechanics Department, The Faculty of Physical Education, Tanta University, Egypt.
}

If we can identify one of kinematics or kinetic characteristics that its improvement is closely related to a similar improvement in technical performance of athletes, from the beginners level to elite performers, this characteristic is called a distinctive indicator. Distinctive indicators of the technical performance efficiency are those indicators distinguishing the improvements in mastering the technical performance of several levels of athletes. Thus, these indicators vary in value depending on the improvements in mastering the technical performance as they appear in distinctive values in the performance of various athletic levels (3).

Performance on the balance beam is characterized with speed and dynamism, increasing the importance of performing in a continuous and flow manner, along with a higher degree of stability. Any trembling or wrong landing may lead to deducting difficulty elements and subtracting tenth of points. This type of performance is characterized with being dangerous and very difficult in initiating and 
flowing the movement, in addition to the possibility of falling off the beam. In short, it represents a hard challenge to the performer (4, $5,6)$.

Performing the motor statement on the balance beam should include five acrobatic elements, with or without hand support, and a flight phase. It is also required to perform forward, backward and sideward acrobatic elements along with aero-circles in all its three forms (straight curved - crouched) as this provides a specific significance for shape and direction. The backward curved aero-circle (Salto backward biked) on the balance beam has a difficulty value of (c) class according to difficulty table. This movement is really important as it is performed either individually or in connection to other preceding or succeeding skills to provide the performer with the linking value ( 7 , 8).

Through monitoring motor statements of performers, it was noted that most performers do not succeed in achieving, mastering or being stable on the balance beam. The researchers think that this is due to the lack of sufficient information during learning and training.

Sports skills, performances and actions all pass through specific phases (preliminary conformity - good conformity - mastering). Each one of these phases has its distinctive characteristics. Changes happening to the performer through motor learning phases are used as indicators to identify the differences among these phases (9, 10). This led the researchers to study these distinctive indicators to identify objective standards and measurements for performance improvement through three levels of performance.These standards and measurements are useful in improving the learning and training processes un gymnastics to achieve the desired athletic levels.

\section{Aims:}

The current research aims at identifying the distinctive indicators of the comparative biomechanical for the Salto backward biked on balance beam three levels of performance through:

1- Identifying the most important biomechanical indicators for the Salto backward biked on balance beam.

2- Designing a educational - training program for the Salto backward biked on balance beam to get junior female gymnastic performers to the mastery phase.

3- Identifying the distinctive indicators of the comparative biomechanical for the Salto backward biked on balance beam three levels of performance .

\section{Hypotheses:}

The researchers hypothesized that:

1. There is some variance in the biomechanical indicators for the Salto backward biked on balance beam.

2. The recommended educational - training program has positive effects on improving the distinctive indicators of the comparative biomechanical for the Salto backward biked on balance beam during three levels of performance Methods:

\section{Approach:}

The researcher used the experimental approach (one-group deign) through follow-up measurements.

\section{Sample:}

Sample (3 junior female gymnastics performers - 11- 12 old year) was purposefully chosen from the Military Institution Sports Club in Alexandria - Egypt. They were all beginners in learning the Salto backward biked skills. 
Table (1)

Sample description

\begin{tabular}{|c|c|c|c|c|c|}
\hline Variables & Measurement & Mean & SD \pm & Median & Squewness \\
\hline Age & Year & 11.7 & 0.13 & 11.66 & -1.04 \\
\hline Height & $\mathrm{Cm}$ & 140.11 & 4.49 & 140.00 & -0.23 \\
\hline Weight & $\mathrm{Kg}$ & 34.89 & 3.70 & 35.00 & 0.267 \\
\hline
\end{tabular}

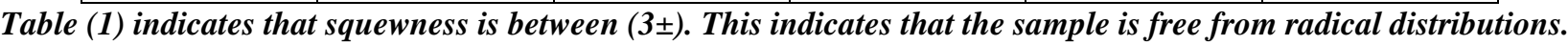

\section{Application:}

Application of the recommended program, along with photographing and measuring were done inside the gymnastics hall of the Military Institution Sports Club in Alexandria - Egypt from 5-6-2010 to 5-8-2010.

\section{Evaluation form: Appendix (1)}

The researchers designed an evaluation form for the Salto backward biked on balance beam three levels of performance according to the following technical points or moments:

1. Maximum fade out moment (through maximum knee bending).
2. Legs- off -the beam moment (vertical push with feet to leave the beam).

3. Flight and circling (in the air).

4. Feet touching the beam (beginning of landing on the beam)

5. Stability and balance moment (end of movement and stability on both feet). (11, $12,13,14)$

The form was presented to experts to dedicate points to each part of the skill, along with nominal points for each phase of. three levels of performance Figure (1) shows the technical performance of the skill.

Fig.1 Technical performance of the Salto backward biked on balance beam

\section{Stability and balance \\ Feet touching the beam}

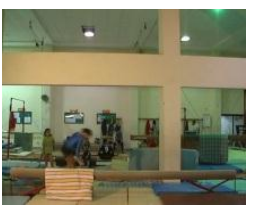

Stability and balance

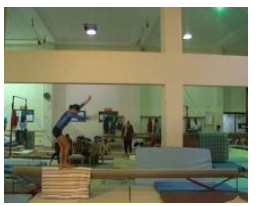

Stability and balance
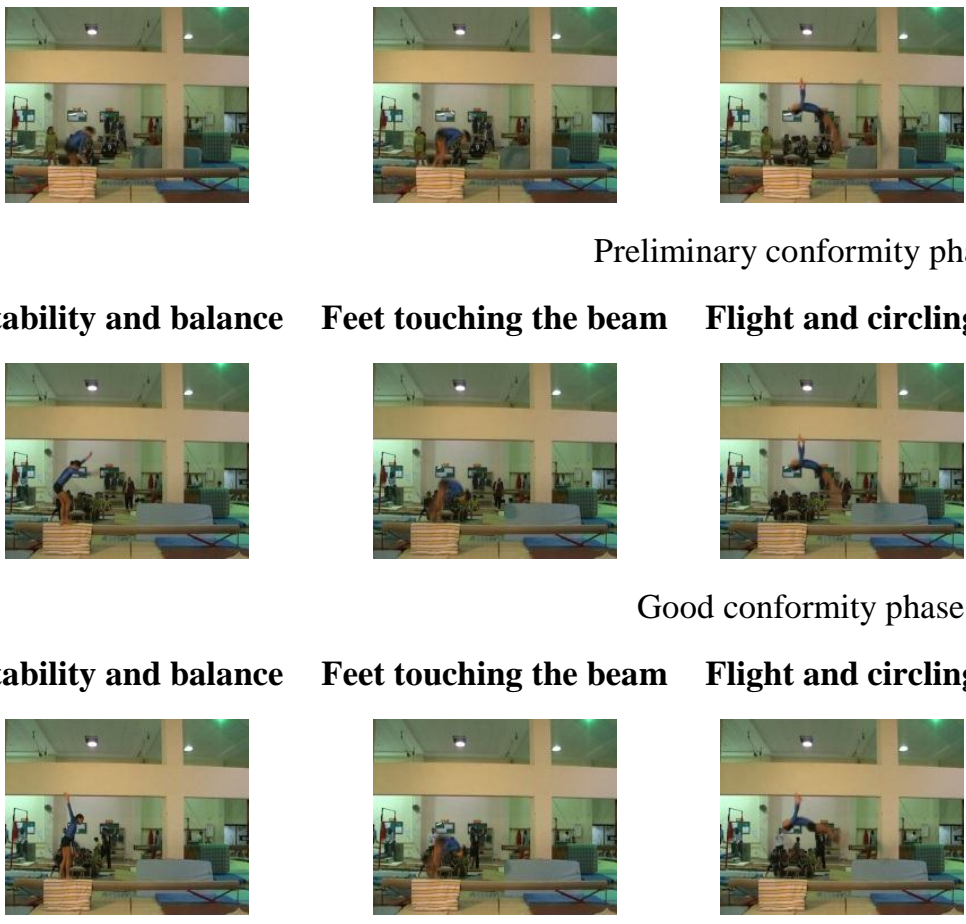

Preliminary conformity phase

Feet touching the beam
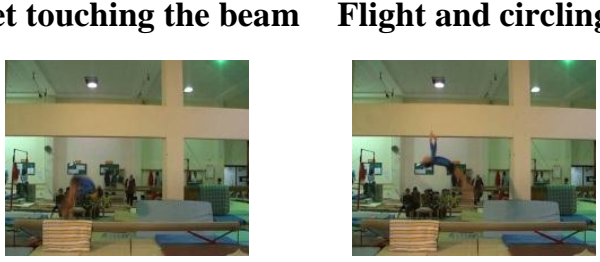

Legs- off -the beam

Good conformity phase

Feet touching the beam

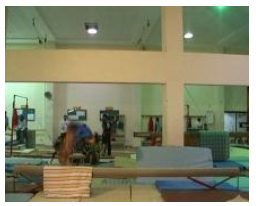

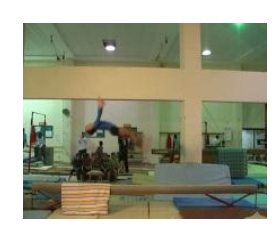

Mastery phase
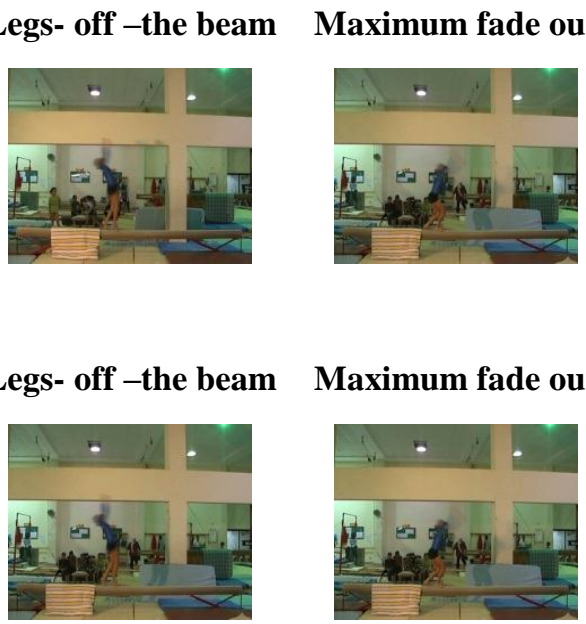

Maximum fade out

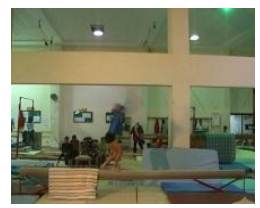

Legs- off -the beam

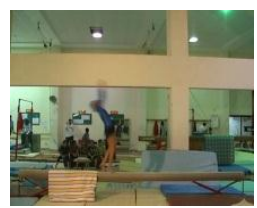

Maximum fade out

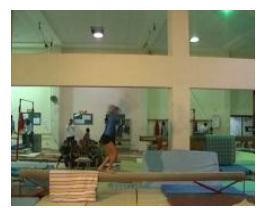




\section{Identification of biomechanical variables:}

Through literature review, the following biomechanical variables are identified as most important for the technical performance of the Salto backward biked skill: leg-foot angle thigh-leg angle - trunk-thigh angle - upper armtrunk angle - upper arm-forearm angle horizontal speed of the body center of gravity vertical speed of the body center of gravity horizontal acceleration of the body center of gravity - vertical acceleration of the body center of gravity - resultant acceleration of the body center of gravity - horizontal momentum - vertical momentum - resultant momentum horizontal strength - vertical strength - resultant strength - altitude of the center of gravity horizontal distance - performance duration. (11, $13,15,16)$

The recommended training program: Appendix (2)

After literature review, the researchers identified the specific exercises used in

Appendix (3) the recommended program. (11, $15,16,17,18)$. Table (2) shows the time distribution of the program.

Table (2)

Time distribution of the recommended program

\begin{tabular}{|c|c|c|c|c|}
\hline Three levels of performance & Weeks & $\begin{array}{c}\text { Training } \\
\text { Units }\end{array}$ & $\begin{array}{c}\text { Unit } \\
\text { Duration }\end{array}$ & Date \\
\hline Preliminary conformity & \multirow{2}{*}{3} & \multirow{2}{*}{12} & \multirow{2}{*}{$50 \mathrm{~min}$} & $5-6 / 23-6-2010$ \\
\hline First follow-up measurement & & & & $24-6-2010$ \\
\hline Good conformity & \multirow{2}{*}{2} & \multirow{2}{*}{8} & \multirow{2}{*}{$50 \mathrm{~min}$} & $26-6 / 7-7-2010$ \\
\hline Second follow-up measurement & & & & $8-7-2010$ \\
\hline Mastery & \multirow{2}{*}{4} & \multirow{2}{*}{16} & \multirow{2}{*}{$50 \min$} & $10-7 / 4-8-2010$ \\
\hline Third follow-up measurement & & & & $5-8-2010$ \\
\hline
\end{tabular}

Follow-up measurements through three levels of performanceprogram:

1. Performers were video-taped and the recorded (CD) was presented to four judges, registered in the Egyptian Federation of Gymnastics, to evaluate the technical performance of each performer in preliminary conformity ( 2.5 to 4 points), good conformity (4.5 to 7 points) and mastery ( 7.5 to 10 points) according to the evaluation form.

2. Performance of each performer was analyzed to identify the biomechanical variables Results: of the Salto backward biked skill on balance beam.

Statistical treatment:

The researchers used the following statistical treatments: mean - standard deviation (SD) median - squewness - multiple regression analysis - variance analyses - Shefee test. Significance was identified on 0.05 for discussion

Table (3)

Multiple Regression equation for choosing performance indicators of the body angles during maximum fade out

$$
\begin{gathered}
\text { Point }=72.218-0.485 \times \text { (upper arm-trunk angle }) \\
R=0.977 \quad R^{2}=0.954
\end{gathered}
$$


Table (4)

Variance analysis among the three performances in the upper arm -trunk angle during maximum fade out

\begin{tabular}{|c|c|c|c|c|c|}
\hline \multirow{2}{*}{ Variable } & Source & $\begin{array}{c}\text { Freedom } \\
\text { Degrees }\end{array}$ & $\begin{array}{c}\text { Sum of } \\
\text { Squares }\end{array}$ & $\begin{array}{c}\text { Mean of } \\
\text { Squares }\end{array}$ & F \\
\hline \multirow{2}{*}{$\begin{array}{c}\text { upper arm - trunk } \\
\text { angle (shoulder angle) }\end{array}$} & Intra-performances & 2 & 143.34 & 71.67 & \multirow{2}{*}{$71.67 *$} \\
\cline { 2 - 6 } & Inter-performances & 6 & 6 & 149.34 & \\
\cline { 2 - 6 } & Total & 8 & & \\
\hline
\end{tabular}

F value on $P \leq 0.05=5.14$

Table (5)

Shefee test for identifying distinctive indicators

\begin{tabular}{|c|c|c|c|c|}
\hline Three Levels of Performance & Mean & $\begin{array}{c}\text { Preliminary } \\
\text { Conformity }\end{array}$ & $\begin{array}{c}\text { Good } \\
\text { Conformity }\end{array}$ & Mastery \\
\hline Preliminary conformity & 142.4 & --- & $-5.9 *$ & $-9.7 *$ \\
\hline Good conformity & 136.5 & & --- & $-3 * 8$ \\
\hline Mastery & 132.7 & & & -- \\
\hline
\end{tabular}

Table (6)

Multiple Regression equation for choosing performance indicators of the body angles during feet-off-the beam moment

Point $=63.577+0.92 \times($ foot-leg angle $)-1.109 \times$ (upper arm-trunk angle $)$

$$
\mathrm{R}=0.977 \quad \mathrm{R}^{2}=0.955
$$

Table (7)

Variance analysis among the three performances in the body angles during feet-off-the beam moment

\begin{tabular}{|c|c|c|c|c|c|}
\hline \multirow{2}{*}{ Variable } & Source & $\begin{array}{c}\text { Freedom } \\
\text { Degrees }\end{array}$ & $\begin{array}{c}\text { Sum of } \\
\text { Squares }\end{array}$ & $\begin{array}{c}\text { Mean of } \\
\text { Squares }\end{array}$ & F \\
\hline \multirow{3}{*}{ Foot-leg angle } & Intra-performances & 2 & 271.04 & 135.520 & \multirow{2}{*}{$135.52^{*}$} \\
\cline { 2 - 5 } & Inter-performances & 6 & 6.00 & 1 & \\
\cline { 2 - 5 } & Total & 8 & 277.040 & & \multirow{3}{*}{$35.56^{*}$} \\
\hline \multirow{3}{*}{ upper arm - trunk angle } & Intra-performances & 2 & 71.12 & 35.56 & \\
\cline { 2 - 5 } & Inter-performances & 6 & 6 & & \\
\cline { 2 - 6 } & Total & 8 & 77.12 & & \\
\hline
\end{tabular}

$F$ value on $P \leq 0.05=5.14$

Table (8)

Shefee test for identifying distinctive indicators

\begin{tabular}{|c|c|c|c|c|}
\hline \multirow{2}{*}{ Variables } & Mean & $\begin{array}{c}\text { Preliminary } \\
\text { Conformity }\end{array}$ & $\begin{array}{c}\text { Good } \\
\text { Conformity }\end{array}$ & Mastery \\
\hline \multirow{3}{*}{ Foot-leg angle } & 106.1 & -- & $8.8^{*}$ & $13.2^{*}$ \\
\cline { 2 - 5 } & 114.9 & & --- & $4.4^{*}$ \\
\cline { 2 - 5 } & 119.3 & & & --- \\
\hline \multirow{3}{*}{ Upper arm-trunk angle } & 142.5 & --- & $5.000^{*}$ & 1.6 \\
\cline { 2 - 5 } & 147.5 & & --- & -- \\
\hline
\end{tabular}


Table (9)

Multiple Regression equation for choosing performance indicators of the body angles during flight and circling phase

Point $=12.642+0.180 \times($ upper arm-trunk angle $)-0.115 \times$ (thigh-leg angle $)$

$$
\mathrm{R}=0.972 \mathrm{R}^{2}=0.944
$$

Table (10)

Variance analysis among the three performances in the body angles during flight and circling phase

\begin{tabular}{|c|c|c|c|c|c|}
\hline Variable & Source & $\begin{array}{c}\text { Freedom } \\
\text { Degrees }\end{array}$ & $\begin{array}{l}\text { Sum of } \\
\text { Squares }\end{array}$ & $\begin{array}{l}\text { Mean of } \\
\text { Squares }\end{array}$ & $\mathbf{F}$ \\
\hline \multirow{3}{*}{$\begin{array}{c}\text { Upper arm - trunk } \\
\text { angle }\end{array}$} & Intra-performances & 2 & 293.329 & 146.664 & \multirow{3}{*}{$146.664 *$} \\
\hline & Inter-performances & 6 & 6.00 & 1 & \\
\hline & Total & 8 & 299.329 & & \\
\hline \multirow{3}{*}{ Thigh-leg angle } & Intra-performances & 2 & 687.62 & 343.81 & \multirow{3}{*}{$343.81 *$} \\
\hline & Inter-performances & 6 & 6.00 & 1 & \\
\hline & Total & 8 & 693.62 & & \\
\hline
\end{tabular}

Table (11)

Shefee test for identifying distinctive indicators

\begin{tabular}{|c|c|c|c|c|c|}
\hline Variables & Performance Phases & Mean & Preliminary conformity & Good conformity & Mastery \\
\hline \multirow{3}{*}{ Upper arm-trunk angle } & Preliminary conformity & 63.29 & -- & $9.68 *$ & $13.58^{*}$ \\
\cline { 2 - 6 } & Good conformity & 72.97 & & --- & $3.9 *$ \\
\cline { 2 - 6 } & Mastery & 76.87 & & $-4.9 *$ & $-20.5 *$ \\
\hline \multirow{3}{*}{ Thigh-leg angle } & Preliminary conformity & 180.4 & & --- & $-15.6 *$ \\
\cline { 2 - 6 } & Good conformity & 175.5 & & & - \\
\cline { 2 - 6 } & Mastery & 159.9 & & & - \\
\hline
\end{tabular}

Table (12)

Multiple Regression equation for choosing performance indicators of the body angles during feet touching the beam moment

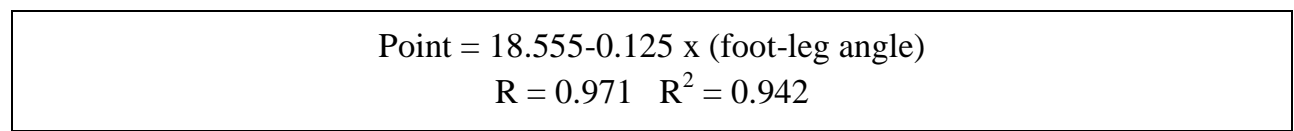

Table (13)

Variance analysis among the three performances in the foot-leg angle during feet touching the beam moment

\begin{tabular}{|c|c|c|c|c|c|}
\hline Variable & Source & Freedom Degrees & Sum of Squares & Mean of Squares & F \\
\hline \multirow{3}{*}{ foot-leg angle } & Intra-performances & 2 & 2192.124 & 1096.062 & \multirow{3}{*}{$359.694^{*}$} \\
\cline { 2 - 5 } & Inter-performances & 6 & 18.283 & 3.047 & \\
\cline { 2 - 6 } & Total & 8 & 2210.408 & & \\
\hline
\end{tabular}

$F$ value on $P \leq 0.05=5.14$ 
Table (14)

Shefee test for identifying distinctive indicators

\begin{tabular}{|c|c|c|c|c|c|}
\hline Variable & $\begin{array}{c}\text { Three Levels of } \\
\text { Performance }\end{array}$ & Mean & $\begin{array}{c}\text { Preliminary } \\
\text { Conformity }\end{array}$ & $\begin{array}{c}\text { Good } \\
\text { Conformity }\end{array}$ & Mastery \\
\hline \multirow{3}{*}{ foot-leg angle } & Preliminary conformity & 120.6 & $---37.853^{*}$ \\
\cline { 2 - 6 } & Good conformity & 106.3 & & -14.3 & -37 \\
\cline { 2 - 6 } & Mastery & 82.747 & & -- & $-23.55 *$ \\
\hline
\end{tabular}

Table (15)

Multiple Regression equation for choosing performance indicators of the body angles during stability and balance moment

$$
\begin{gathered}
\text { Point }=2.670+0.030 \times(\text { thigh-trunk angle }) \\
R=0.904 \quad R^{2}=0.817
\end{gathered}
$$

\section{Table (16)}

Variance analysis among the three performances in the foot-leg angle during stability and balance moment

\begin{tabular}{|c|c|c|c|c|c|}
\hline Variable & Source & Freedom degrees & Sum of squares & Mean of squares & F \\
\hline \multirow{3}{*}{ thigh-trunk angle } & Intra-performances & 1 & 8916.615 & 8916.615 & \multirow{3}{*}{$3566.646^{*}$} \\
\cline { 2 - 6 } & Inter-performances & 4 & 10.00 & 2.5 & \\
\cline { 2 - 6 } & \multicolumn{7}{|c|}{ Total } & 5 & 8926.615 & & \\
\hline
\end{tabular}

Table (17)

Shefee test for identifying distinctive indicators

\begin{tabular}{|c|c|c|c|c|c|}
\hline Variable & Three Levels of Performance & Mean & $\begin{array}{c}\text { Preliminary } \\
\text { Conformity }\end{array}$ & $\begin{array}{c}\text { Good } \\
\text { Conformity }\end{array}$ & Mastery \\
\hline \multirow{2}{*}{$\begin{array}{c}\text { thigh-trunk } \\
\text { angle }\end{array}$} & Preliminary conformity & --- & --- & & \\
\cline { 2 - 6 } & Good conformity & 100.2 & & --- & -- \\
\cline { 2 - 6 }
\end{tabular}

Table (18)

Multiple Regression equation for choosing performance indicators of the body center of gravity during maximum fade out moment

Point $=9.882-2.135 \times$ (horizontal acceleration) $-0.115 \times$ (resultant speed $)$

$$
\mathrm{R}=0.980 \mathrm{R}^{2}=0.961
$$

Point $=18.027-0.103 \mathrm{x}($ horizontal strength $)-0.115 \mathrm{x}$ (resultant strength)

$$
\mathrm{R}=0.977 \mathrm{R}^{2}=0.954
$$


Table (19)

Variance analysis among the three performances in the body center of gravity during maximum fade out moment

\begin{tabular}{|c|c|c|c|c|c|}
\hline Variable & Source & Freedom Degrees & Sum of Squares & Mean of Squares & $\mathbf{F}$ \\
\hline \multirow{3}{*}{$\begin{array}{l}\text { Horizontal } \\
\text { acceleration }\end{array}$} & Intra-performances & 2 & 7.249 & 3.625 & \multirow{3}{*}{$483.276^{*}$} \\
\hline & Inter-performances & 6 & 0.045 & 0.0075 & \\
\hline & Total & 8 & 7.294 & & \\
\hline \multirow{3}{*}{$\begin{array}{l}\text { Resultant } \\
\text { speed }\end{array}$} & Intra-performances & 2 & 0.291 & 0.146 & \multirow{3}{*}{$30.11 *$} \\
\hline & Inter-performances & 6 & 0.0290 & 0.0048 & \\
\hline & Total & 8 & 0.320 & & \\
\hline \multirow{3}{*}{$\begin{array}{l}\text { Horizontal } \\
\text { strength }\end{array}$} & Intra-performances & 2 & 7889.077 & 3944.539 & \multirow{3}{*}{$39445.387 *$} \\
\hline & Inter-performances & 6 & 0.600 & 0.100 & \\
\hline & Total & 8 & 7889.677 & & \\
\hline \multirow{3}{*}{$\begin{array}{l}\text { Resultant } \\
\text { strength }\end{array}$} & Intra-performances & 2 & 2146.88 & 1073.44 & \multirow{3}{*}{$1073.44 *$} \\
\hline & Inter-performances & 6 & 6.00 & 1.00 & \\
\hline & Total & 8 & 2152.88 & & \\
\hline
\end{tabular}

Table (20)

Shefee test for identifying distinctive indicators

\begin{tabular}{|c|c|c|c|c|c|}
\hline Variables & Performance Phases & Mean & $\begin{array}{l}\text { Preliminary } \\
\text { Conformity }\end{array}$ & Good Conformity & Mastery \\
\hline \multirow{3}{*}{$\begin{array}{c}\text { Horizontal } \\
\text { acceleration }\end{array}$} & Preliminary conformity & -0.455 & --- & $-0.461 *$ & $-2.092 *$ \\
\hline & Good conformity & -0.916 & & -- & $-1.631 *$ \\
\hline & Mastery & -2.547 & & & --- \\
\hline \multirow{3}{*}{ Resultant speed } & Preliminary conformity & 2.048 & --- & $-0.422 *$ & -0.101 \\
\hline & Good conformity & 1.626 & & --- & $0.321 *$ \\
\hline & Mastery & 1.947 & & & --- \\
\hline \multirow{3}{*}{ Horizontal strength } & Preliminary conformity & -15.03 & --- & $-15.23 *$ & $-61.02 *$ \\
\hline & Good conformity & 30.26 & & --- & $-53.79 *$ \\
\hline & Mastery & -84.05 & & & --- \\
\hline \multirow{3}{*}{ Resultant strength } & Preliminary conformity & 187 & --- & $-10.8^{*}$ & $26^{*}$ \\
\hline & Good conformity & 176.2 & & --- & $36.8 *$ \\
\hline & Mastery & 213 & & & --- \\
\hline
\end{tabular}

Table (21)

Multiple Regression equation for choosing performance indicators of the body center of gravity during feet-off-the beam moment

Point $=17.896-5.373 \times$ (resultant speed $)$

$$
\mathrm{R}=0.818 \mathrm{R}^{2}=0.670
$$

Point $=11.374-2.558 \mathrm{x}($ vertical momentum $)+2.236 \mathrm{x}$ (resultant momentum $)$

$$
\mathrm{R}=0.975 \mathrm{R}^{2}=0.951
$$


Table (22)

Variance analysis among the three performances in the body center of gravity during feet-off-the beam moment

\begin{tabular}{|c|c|c|c|c|c|}
\hline Variable & Source & Freedom Degrees & Sum of Squares & Mean of Squares & $\mathbf{F}$ \\
\hline \multirow{3}{*}{ Resultant speed } & Intra-performances & 2 & 0.796 & 0.398 & \multirow{3}{*}{$39.78^{*}$} \\
\hline & Inter-performances & 6 & 0.0600 & 0.0100 & \\
\hline & Total & 8 & 0.856 & & \\
\hline \multirow{3}{*}{$\begin{array}{c}\text { Vertical } \\
\text { momentum }\end{array}$} & Intra-performances & 2 & 667.197 & 333.598 & \multirow{3}{*}{$333.598 *$} \\
\hline & Inter-performances & 6 & 6 & 1 & \\
\hline & Total & 8 & 673.197 & & \\
\hline \multirow{3}{*}{$\begin{array}{l}\text { Resultant } \\
\text { momentum }\end{array}$} & Intra-performances & 2 & 757.261 & 378.63 & \multirow{3}{*}{$378.63 *$} \\
\hline & Inter-performances & 6 & 6 & 1 & \\
\hline & Total & 8 & 763.261 & & \\
\hline
\end{tabular}

F value on $P \leq 0.05=5.14$

Table (23)

Shefee test for identifying distinctive indicators

\begin{tabular}{|c|c|c|c|c|c|}
\hline Variables & Performance Phases & Mean & $\begin{array}{l}\text { Preliminary } \\
\text { Conformity }\end{array}$ & Good Conformity & Mastery \\
\hline \multirow{3}{*}{ Resultant speed } & Preliminary conformity & 2.487 & --- & 0.019 & -0.621 \\
\hline & Good conformity & 2.506 & & --- & -.064 \\
\hline & Mastery & 1.866 & & & --- \\
\hline \multirow{3}{*}{ Vertical momentum } & Preliminary conformity & 63.17 & --- & 2.46 & $-16.91 *$ \\
\hline & Good conformity & 65.63 & & --- & $-19.37 *$ \\
\hline & Mastery & 46.26 & & & --- \\
\hline \multirow{3}{*}{ Resultant momentum } & Preliminary conformity & 78.77 & --- & $3.92 *$ & $-17.2 *$ \\
\hline & Good conformity & 82.69 & & --- & $-21.12 *$ \\
\hline & Mastery & 61.57 & & & --- \\
\hline
\end{tabular}

Table (24)

Multiple Regression equation for choosing performance indicators of the body center of gravity during flight and circling phase

$$
\begin{gathered}
\text { Point }=28.114-5.815 \times \text { (horizontal acceleration }) \\
R=0.961 \mathrm{R}^{2}=0.924 \\
\text { Point }=5.455+0.328 \times(\text { resultant momentum }) \\
R=0.964 \mathrm{R}^{2}=0.930
\end{gathered}
$$


Table (25)

Variance analysis among the three performances in the body center of gravity during flight and circling phase

\begin{tabular}{|c|c|c|c|c|c|}
\hline Variable & Source & $\begin{array}{c}\text { Freedom } \\
\text { Degrees }\end{array}$ & $\begin{array}{c}\text { Sum of } \\
\text { Squares }\end{array}$ & $\begin{array}{l}\text { Mean of } \\
\text { Squares }\end{array}$ & $\mathbf{F}$ \\
\hline \multirow{3}{*}{$\begin{array}{c}\text { Horizontal } \\
\text { acceleration }\end{array}$} & $\begin{array}{c}\text { Intra- } \\
\text { performances }\end{array}$ & 2 & 0.987 & 0.494 & \multirow{3}{*}{$144.917 *$} \\
\hline & $\begin{array}{c}\text { Inter- } \\
\text { performances }\end{array}$ & 6 & 0.02044 & 0.0034 & \\
\hline & Total & 8 & 1.008 & & \\
\hline \multirow{3}{*}{$\begin{array}{l}\text { Resultant } \\
\text { momentum }\end{array}$} & $\begin{array}{c}\text { Intra- } \\
\text { performances }\end{array}$ & 2 & 319.756 & 159.878 & \multirow{3}{*}{$22948.981 *$} \\
\hline & $\begin{array}{c}\text { Inter- } \\
\text { performances }\end{array}$ & 6 & 0.0418 & 10.006967 & \\
\hline & Total & 8 & 319.798 & & \\
\hline
\end{tabular}

Table (26)

Shefee test for identifying distinctive indicators

\begin{tabular}{|c|c|c|c|c|c|}
\hline Variables & Performance Phases & Mean & $\begin{array}{c}\text { Preliminary } \\
\text { Conformity }\end{array}$ & Good Conformity & Mastery \\
\hline \multirow{3}{*}{$\begin{array}{c}\text { Horizontal } \\
\text { acceleration }\end{array}$} & Preliminary conformity & 4.311 & --- & $-0.526^{*}$ & $-0.798^{*}$ \\
\cline { 2 - 6 } & Good conformity & 3.785 & & --- & $-0.272^{*}$ \\
\cline { 2 - 6 } & Mastery & 3.513 & & --- \\
\hline \multirow{2}{*}{$\begin{array}{c}\text { Resultant } \\
\text { momentum }\end{array}$} & Preliminary conformity & 27.03 & --- & $5.77^{*}$ & $14.5^{*}$ \\
\cline { 2 - 6 } & Good conformity & 32.8 & & --- & $8.73^{*}$ \\
\cline { 2 - 6 } & Mastery & 41.53 & & -- \\
\hline
\end{tabular}

Table (27)

Multiple Regression equation for choosing performance indicators of the body center of gravity during feet touching the beam phase

Point $=6.886+0.932 \times$ (horizontal acceleration)

$$
\mathrm{R}=0.915 \mathrm{R}^{2}=0.836
$$

Point $=53.597+1.743 \times$ (horizontal momentum)

$$
\mathrm{R}=0.942 \mathrm{R}^{2}=0.888
$$

Table (28)

\begin{tabular}{|c|c|c|c|c|c|}
\hline Variable & Source & Freedom Degrees & Sum of Squares & Mean of Squares & $\mathbf{F}$ \\
\hline \multirow{3}{*}{$\begin{array}{c}\text { Horizontal } \\
\text { acceleration }\end{array}$} & Intra-performances & 1 & 9.42 & 9.42 & \multirow{3}{*}{$942.005 *$} \\
\hline & Inter-performances & 4 & 0.0400 & 0.0100 & \\
\hline & Total & 5 & 9.46 & & \\
\hline \multirow{3}{*}{$\begin{array}{l}\text { Horizontal } \\
\text { momentum }\end{array}$} & Intra-performances & 2 & 17.79 & 8.895 & \multirow{3}{*}{$190.603 *$} \\
\hline & Inter-performances & 6 & 0.280 & 0.04667 & \\
\hline & Total & 8 & 18.070 & & \\
\hline
\end{tabular}

Variance analysis among the three performances in the body center of gravity during feet touching the beam phase 
Table (29)

Shefee test for identifying distinctive indicators

\begin{tabular}{|c|c|c|c|c|c|}
\hline \multirow{2}{*}{ Variables } & Performance Phases & Mean & $\begin{array}{c}\text { Preliminary } \\
\text { Conformity }\end{array}$ & Good Conformity & Mastery \\
\hline \multirow{4}{*}{ Horizontal acceleration } & Preliminary conformity & & & & \\
\cline { 2 - 6 } & Good conformity & -1.31 & & & \\
\cline { 2 - 6 } & Mastery & 1.196 & & $-3.42^{*}$ & $-2.06^{*}$ \\
\hline \multirow{3}{*}{ Horizontal momentum } & Preliminary conformity & -24.09 & --- & -- & $1.36^{*}$ \\
\cline { 2 - 6 } & Good conformity & -27.51 & & & --- \\
\cline { 2 - 6 } & Mastery & -26.15 & & & \\
\hline
\end{tabular}

Table (30)

Multiple Regression equation for choosing performance indicators of the body center of gravity during stability and balance phase

Point $=8.081-0.158 \times$ (vertical momentum $)$
$\mathrm{R}=0.926 \mathrm{R}^{2}=0.858$
Point $=8.396-1.179 \times$ (resultant acceleration)
$\mathrm{R}=0.924 \mathrm{R}^{2}=0.854$

Table (31)

Variance analysis among the three performances in the body center of gravity during stability and balance phase

\begin{tabular}{|c|c|c|c|c|c|}
\hline Variable & Source & Freedom Degrees & Sum of Squares & Mean of Squares & F \\
\hline \multirow{3}{*}{$\begin{array}{c}\text { Vertical } \\
\text { momentum }\end{array}$} & Intra-performances & 1 & 333.865 & 333.865 & \multirow{2}{*}{$667.649 *$} \\
\cline { 2 - 6 } & Inter-performances & 4 & 2 & 0.5 & \\
\cline { 2 - 5 } & Total & 5 & 335.865 & & \\
\multirow{2}{*}{$\begin{array}{c}\text { Resultant } \\
\text { acceleration }\end{array}$} & Intra-performances & 1 & 6.024 & 6.024 \\
\cline { 2 - 6 } & Inter-performances & 4 & 0.0211 & 0.00529 \\
\cline { 2 - 6 } & Total & 5 & 6.045 & & \\
\hline
\end{tabular}

$F$ value on $P \leq 0.05=5.14$

Table (32)

Shefee test for identifying distinctive indicators

\begin{tabular}{|c|c|c|c|c|c|}
\hline \multirow{2}{*}{ Variables } & Performance Phases & Mean & $\begin{array}{c}\text { Preliminary } \\
\text { Conformity }\end{array}$ & Good Conformity & Mastery \\
\hline \multirow{3}{*}{ Horizontal acceleration } & Preliminary conformity & & --- & & \\
\cline { 2 - 6 } & Good conformity & 15.33 & & -- & -- \\
\cline { 2 - 6 } & Mastery & 0.411 & & & \\
\hline \multirow{3}{*}{ Resultant momentum } & Preliminary conformity & & --- & -- & - \\
\cline { 2 - 6 } & Good conformity & 2.328 & & & - \\
\cline { 2 - 6 } & Mastery & 0.324 & & & \\
\hline
\end{tabular}


Table (33)

Multiple Regression equation for choosing performance indicators through learning phases for horizontal distance, center of gravity altitude and performance duration

Point $=0.287-27.042 \times($ horizontal distance $)+22.420($ center of gravity altitude $)$

$$
\mathrm{R}=0.907 \mathrm{R}^{2}=0.822
$$

Point $=2.659+4.744($ final phase duration $)$

$$
\mathrm{R}=0.967 \mathrm{R}^{2}=0.936
$$

Table (34)

Variance analysis among the three performances in the for horizontal distance, center of gravity altitude and

\begin{tabular}{|c|c|c|c|c|c|}
\hline Variable & Source & $\begin{array}{c}\text { Freedom } \\
\text { Degrees }\end{array}$ & $\begin{array}{c}\text { Sum of } \\
\text { Squares }\end{array}$ & $\begin{array}{l}\text { Mean of } \\
\text { Squares }\end{array}$ & $\mathbf{F}$ \\
\hline \multirow{3}{*}{ Horizontal distance } & Intra-performances & 2 & 0.0222 & 0.0111 & \multirow{3}{*}{$8.1228 *$} \\
\hline & Inter-performances & 6 & 0.0082 & 0.00136 & \\
\hline & Total & 8 & 0.0304 & & \\
\hline \multirow{3}{*}{$\begin{array}{c}\text { Center of gravity } \\
\text { altitude }\end{array}$} & Intra-performances & 2 & 0.0042 & 0.0021 & \multirow{3}{*}{1.26} \\
\hline & Inter-performances & 6 & 0.01 & 0.00166 & \\
\hline & Total & 8 & 0.0142 & & \\
\hline \multirow{3}{*}{$\begin{array}{l}\text { Final phase } \\
\text { duration }\end{array}$} & Intra-performances & 2 & 1.524 & 0.762 & \multirow{3}{*}{$451.132 *$} \\
\hline & Inter-performances & 6 & 0.0101 & 0.00168 & \\
\hline & Total & 8 & 1.534 & & \\
\hline
\end{tabular}
performance duration

Table (35)

\begin{tabular}{|c|c|c|c|c|c|}
\hline Variables & Performance Phases & Mean & $\begin{array}{l}\text { Preliminary } \\
\text { Conformity }\end{array}$ & $\begin{array}{c}\text { Good } \\
\text { Conformity }\end{array}$ & Mastery \\
\hline \multirow{3}{*}{ Horizontal distance } & Preliminary conformity & 0.81 & --- & $-0.100 *$ & $-0.110 *$ \\
\hline & Good conformity & 0.71 & & --- & -0.01 \\
\hline & Mastery & 0.70 & & & --- \\
\hline \multirow{3}{*}{ Center of gravity altitude } & Preliminary conformity & 1.100 & --- & & \\
\hline & Good conformity & 1.1400 & & --- & \\
\hline & Mastery & 1.1500 & & & -- \\
\hline \multirow{3}{*}{ Final phase duration } & Preliminary conformity & 0.1333 & --- & $0.46^{*}$ & $1.0067 *$ \\
\hline & Good conformity & 0.5933 & & --- & $0.5467 *$ \\
\hline & Mastery & 1.140 & & & --- \\
\hline
\end{tabular}

Shefee test for identifying distinctive indicators

\section{Discussion:}

Tables (3-6-9-12-15-18-21-24-27-30-33) are concerned with turning biomechanical variables of the skill into biomechanical indicators related to moments of technical performance. Using multiple regression equation the following indicators of body angles are concluded: legfoot angle - thigh-leg angle - trunk-thigh angle - upper arm-trunk angle - upper arm-forearm angle. Body center of gravity indicators are as follows: horizontal acceleration - resultant speed - horizontal strength - resultant strength - 
horizontal momentum - vertical momentum resultant momentum - resultant acceleration. Other indicators are: horizontal distance center of gravity altitude - final phase duration.

Tables

(4-7-10-13-16-19-22-25-28-31-34)

showed differences among biomechanical indicators according to the motor learning phases, using variance analysis. All variable (F values between 35.56 and 39445.378) are significant in favor of mastery phase, except for center of gravity altitude as (F) value was 1.26.

Tables (5-8-11-14-17-20-23-26-29-32-35), using Sheffee test, showed the distinctive indicators comparing the three levels of performance related to moments of performance. As for Maximum fade out moment, distinctive biomechanical indicators are upper arm- forearm angle, horizontal acceleration, horizontal strength and resultant strength as they differentiated the three phases in favor of the mastery phase. Only resultant speed is not a distinctive indicator as it did not differentiate among the three phases. As for feet-off-the beam moment, distinctive biomechanical indicators are foot-leg angle and resultant momentum, while upper arm-forearm angle, resultant speed and vertical momentum are only normal indicators as they did not differentiate among the three phases.

After maximum fade out moment for bending knees, bent legs are stretched with pushing from the spine to give the body maximum elevation during feet-off the beam moment. Forces resulting in circular movements during flight begin before the performer leaves the beam surface. Biomechanical distinctive indicators for flight and circling moment are upper arm-trunk angle, thigh-leg angle, horizontal acceleration and resultant momentum as they differentiated among the three phases in favor of the mastery phase. At this moment, trunk and arms begin circling heavily due to arm braking at maximum elevation. This transfers momentum to lower limbs to elevated the upper body. Thus, thigh joint speed increases to shorten the circling diameter and the upper and lower parts of the body come closer to form the curved shape needed for circling.

As for feet-touching- the beam moment, distinctive biomechanical indicators are foot-leg angle and horizontal momentum, as they differentiated among the three phases in favor of the mastery phase. Horizontal acceleration was considered a normal indicator as it differentiated between good conformity and mastery phases.

\section{Conclusions:}

The researcher concluded the following distinctive biomechanical indicators to differentiated among the three levels of performance (preliminary conformity - good conformity - master):

1. Maximum fade out distinctive indicators are upper arm- forearm angle, horizontal acceleration, horizontal strength and resultant strength.

2. Feet-off the beam distinctive indicators are foot-leg angle and resultant momentum.

3. Flight and circling distinctive indicators are upper arm-trunk angle, thigh-leg angle, horizontal acceleration and resultant momentum.

4. Feet-touching-the beam distinctive indicators are foot-leg angle and horizontal momentum.

5. Stability and balance distinctive indicators are thigh-trunk angle, vertical momentum and resultant acceleration.

6. Duration of performance for final phase.

7. Predictive equations indicating the performance level of the Salto backward biked 
on the balance beam during motor learning phases.

\section{Recommendations:}

The researchers recommend the following:

1. Using distinctive indicators to evaluate the biomechanical efficiency of performing Salto backward biked on the balance beam.

2. Using the concluded predictive equations in measuring the performance improvement of Salto backward biked on the balance beam during three levels of performance

3. Coaches should study basics of biomechanics and motor analysis to solve technical performance problems for gymnastic performers.

\section{References:}

1. Alaa El-Din, Gamal\& Al-Sabbagh, Nahed A. (2007): Kinematics, $9^{\text {th }}$ ED, Dar AlKetab, Alexandria - Egypt, P:3 (in Arabic).

2. Carhgenny (1997): Mechanics of sport human kinetics. Gerland A. Carr USA. P:3

3. Alaa El-Din, Gamal\& Al-Sabbagh, Nahed A. (2007): Metrological Bases for evaluating physical, technical and tactical performance for athletes. Monshaat AlMaaref, Alexandria - Egypt, PP: 55, 66 (in Arabic).

4. Afify, Sherifa A. (2003): Effectiveness of balance excursuses and its effects on some movement chains and performance level on the balance beam for gymnastic performers less than 10 years. He scientific journal, Faculty of Physical Education - Tanta University - Egypt. P:63 (in Arabic)

5. Farouz, Sabah \&Shehata, Mohamed E. (2007); Gymnastics Guide for female performers. The Egyptian Library Press, PP:10, 12 (in Arabic).
6. Abd El-Rahman, Aziza \& Salem, Aziza M. (2001): Gymnastics between theory and application. Markaz Al-Ketab Press, Cairo - Egypt. PP:115-116, 233. in Arabic)

7. Women's artistic gymnastics: International gymnastics federation of paints, $12^{\text {th }}$ cycle, 2009. PP:115-116, 233.

8. Shehata, Mohamed E. \& Al-Shazly, Ahmed F. (2006): Field applications for motor analysis in gymnastics. The Egyptian Library, $1^{\text {st }}$ ED - Alexandria - Egypt. P:75 (in Arabic).

9. Counliman, J. E. (1980): The complete book of competitive swimming, New York, USA. P:214.

10. Magill, Richard A. (2001): Motor learning concepts $\&$ applications, $6^{\text {th }} \mathrm{ED}$, McGrawHill book Co., Boston. P:188.

11. Hodoud, Khaled M. (Biomechanical indicators as a basis for designing specific exercises to improve the technical performance of front and Salto backward biked for junior gymnastic performers. PhD thesis, Faculty of Physical Education for Men - Alexandria - Egypt. PP:15-16, 4950 (in Arabic).

12. Shehata, Ahamed E. \& Al-Shazly, Ahmed F. (1986): Floor movements in gymnastics. The Fannia Press, Alexandria - Egypt. P:110 (in Arabic).

13. Shehata, Mohamed E. (1991): Motor analysis in gymnastics. Al-Touny Press, Alexandria - Egypt. P:119-120, 92-94 (in Arabic).

14. Price, H. D. (1974): Gymnastics and tumbling, Arco Publishing Company INC, New York. P:32

15. Heliel, Hatem A. \& Al-Shamy, Mohamed A. (2007): Biomechanical indicators as a 
basis for directing specific training of the back double SALTO skill from the preparation skill on floor. Second international conference, Faculty of Physical Education for Men - Zagazig University, Vol.2, P:335. (in Arabic).

16. Ahmed, Mohab A. (2002): Effects of specific training of kinesthetic cognition on improving the front somersault performance on balance beam. PhDthesis, Faculty of Physical Education for Men Alexandria - Egypt. PP: 43-44 (in Arabic)
17. Al-Hagrasy, Abd El-Raouf A. \&Hussain, Hadiat A. (2008): Training Rules in Gymnastics. Unpublished university notes. Faculty of Physical Education for Women Helwan University - Egypt. PP: 28, 38-56 (in Arabic).

18. Abd El-Aal, Soha M (2001): Identifying the kinematics contributing in Salto backward biked performance for landing on the balance beam. Master thesis, Faculty of Physical Education for Women Alexandria - Egypt. P: 89-99 (in Arabic). 